\title{
Cortical Networks Generating Movement-Related EEG Rhythms in Alzheimer's Disease: An EEG Coherence Study
}

\author{
Claudio Babiloni \\ Università degli Studi di Roma "La Sapienza," Istituto di \\ Ricovero e Cura a Carattere Scientifico "S. Giovanni di Dio \\ Fatebenefratelli," and A. Fa. R., Ospedale Fatebenefratelli \\ Isola Tiberina \\ Davide V. Moretti \\ Università degli Studi di Roma "La Sapienza," A. Fa. R., \\ Ospedale Fatebenefratelli Isola Tiberina, and Istituto di Ricovero \\ e Cura a Carattere Scientifico "S. Giovanni \\ di Dio Fatebenefratelli" \\ Serenella Salinari \\ Università degli Studi di Roma "La Sapienza"
}

\author{
Carlo Miniussi \\ Istituto di Ricovero e Cura a Carattere Scientifico "S. Giovanni \\ di Dio Fatebenefratelli" \\ Fabrizio Vecchio \\ Università degli Studi di Roma "La Sapienza" and A. Fa. R., \\ Ospedale Fatebenefratelli Isola Tiberina
}

\section{Giovanni Frisoni}

Istituto di Ricovero e Cura a Carattere Scientifico "S. Giovanni di Dio Fatebenefratelli” and A. Fa. R., Ospedale Fatebenefratelli Isola Tiberina

Paolo Maria Rossini

Istituto di Ricovero e Cura a Carattere Scientifico "S. Giovanni di Dio Fatebenefratelli," Università degli Studi di Roma Campus Biomedico, and A. Fa. R., Ospedale Fatebenefratelli Isola Tiberina

\begin{abstract}
Patients with mild Alzheimer's disease (AD) present with abnormally strong values of frontal and ipsilateral central sensorimotor rhythms. The authors tested 2 working hypotheses of the related electroencephalographic (EEG) coherence: disconnection, defined as a sign of a reduced coordination within the frontoparietal and interhemispheric networks, and cooperation, defined as a reflection of the reorganization of the brain sensorimotor networks. Results showed that, compared with healthy controls, patients with mild AD had an unreactive and abnormally low interhemispheric EEG coherence and an unreactive and abnormally high frontoparietal EEG coherence. These findings support the hypothesis of an impaired mechanism of sensorimotor cortical coupling (disconnection) in mild AD.
\end{abstract}

Alzheimer's disease (AD) is a neurodegenerative disease characterized by a progressive loss of cognitive functions. In mild to moderate $\mathrm{AD}$, negligible or no sensorimotor symptoms are observed, such as a subclinical slowing of movement time (Goldman,
Baty, Buckles, Sahrmann, \& Morris, 1999). To investigate the cortical substrate of the motor performances in patients with early $\mathrm{AD}, \mathrm{C}$. Babiloni et al. (2000) recently modeled their sensorimotor cortical electroencephalographic (EEG) rhythmicity. It was found
Claudio Babiloni, Dipartimento Fisiologia Umana e Farmacologia, Università degli Studi di Roma "La Sapienza," Rome; Istituto di Ricovero e Cura a Carattere Scientifico "S. Giovanni di Dio Fatebenefratelli," Brescia, Italy; and Associaciazione Fatebenefratelli per la Ricerca (A. Fa. R.), Ospedale Fatebenefratelli Isola Tiberina, Rome. Carlo Miniussi, Istituto di Ricovero e Cura a Carattere Scientifico "S. Giovanni di Dio Fatebenefratelli." Davide V. Moretti, Dipartimento Fisiologia Umana e Farmacologia, Università degli Studi di Roma "La Sapienza"; A. Fa. R., Ospedale Fatebenefratelli Isola Tiberina; and Istituto di Ricovero e Cura a Carattere Scientifico "S. Giovanni di Dio Fatebenefratelli." Fabrizio Vecchio, Dipartimento Fisiologia Umana e Farmacologia, Università degli Studi di Roma "La Sapienza"; and A. Fa. R., Ospedale Fatebenefratelli Isola Tiberina. Serenella Salinari, Dipartimento di Informatica e Sistemistica, Università degli Studi di Roma "La Sapienza." Giovanni Frisoni, Istituto di Ricovero e Cura a Carattere Scientifico "S. Giovanni di Dio Fatebene- fratelli"; and A. Fa. R., Ospedale Fatebenefratelli Isola Tiberina. Paolo Maria Rossini, Istituto di Ricovero e Cura a Carattere Scientifico "S. Giovanni di Dio Fatebenefratelli"; Clinica Neurologica, Università degli Studi de Roma Campus Biomedico, Rome; and A. Fa. R., Ospedale Fatebenefratelli Isola Tiberina.

This research was supported by the Telethon Onlus Foundation ("Progetto E.C0985") and A. Fa. R. We thank Orazio Zanetti and Giuliano Binetti for their contributions to the selection of the patients with Alzheimer's disease and the healthy subjects. We also thank Fabrizio Eusebi for his continuous support.

Correspondence concerning this article should be addressed to Claudio Babiloni, Dipartimento Fisiologia Umana e Farmacologia, Sezione di EEG ad Alta Risoluzione, Università degli Studi di Roma "La Sapienza," Piazzale Aldo Moro 5, 00185 Rome, Italy. E-mail: claudio.babiloni@, uniromal.it 

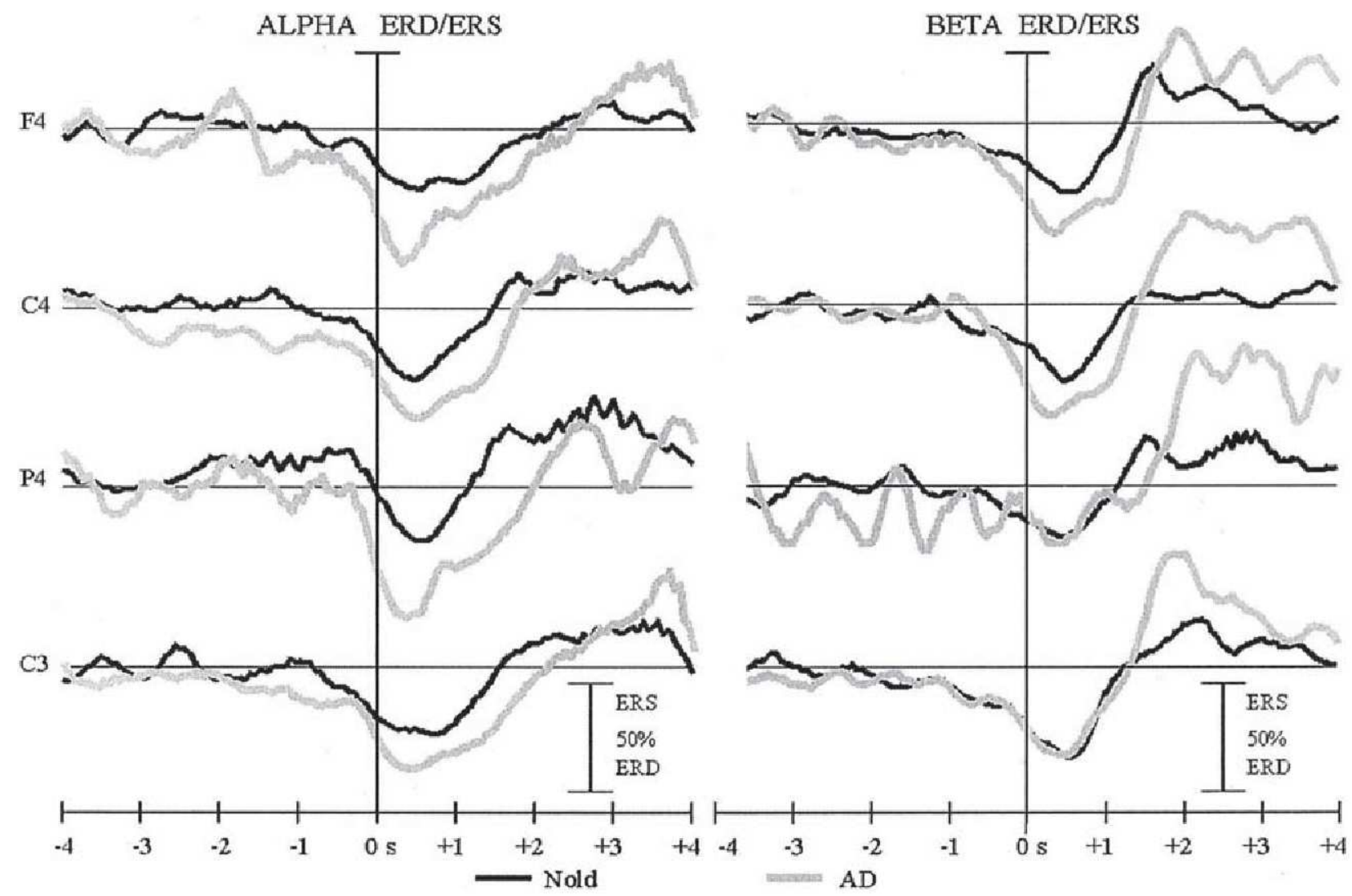

Figure 1. Grand average waveforms of alpha and beta event-related desynchronization/synchronization (ERD/ ERS), computed from frontal, central, and parietal electrode sites ( $\mathrm{F} 4, \mathrm{C} 4, \mathrm{P} 4)$ ipsilateral to the movement in patients with Alzheimer's disease (AD) and age-matched control subjects ("normal old"; Nold). The grand average waveforms of alpha and beta ERD/ERS computed from contralateral (C3) electrodes are also illustrated as a reference. The ERD/ERS peaks were computed from Laplacian-transformed electroencephalographic (EEG) potentials recorded from 19 channels (standard 10-20 system). The motor task consisted of an externally triggered extension of the right middle finger. The procedure for the ERD/ERS computation was based on the standard methodology used by the Graz group: (a) preliminary bandpass filtering of EEG single trials (alpha or beta band), (b) squaring of amplitude values, (c) averaging of the amplitude in time and across trials, and (d) computation of the percent increase or decrease of the squared amplitude during the movement or postmovement referenced to a premovement baseline period.

that motor performance and event-related desynchronization/synchronization of EEG oscillations (ERD/ERS; Pfurtscheller, Neuper, Andrew, \& Edlinger, 1997; Pfurtscheller, Pichler-Zalaudek, \& Neuper, 1999) were preserved over rolandic cortex contralateral to the movement (see Figure 1). In contrast, patients' postmovement frontal ERS was abnormally high at the alpha and beta bands $(p<$ $.05)$, whereas the beta postmovement central ERS was greater only at the ipsilateral hemisphere $(p<.05)$. These findings suggest that the cortical processing of sensorimotor information is affected at a preclinical level in early $\mathrm{AD}$. However, it is still questionable whether the abnormally enhanced frontal and ipsilateral central EEG activity was due to either impaired (i.e., "disconnection") or enhanced ("cooperation" for deficit compensation) neural networks subserving the motor control.

To address this issue, the present study modeled the sensorimotor cortical coupling from the same EEG data set showing abnormal ERD/ERS in patients with early AD performing simple finger movements (C. Babiloni et al., 2000). The frontoparietal and interhemispheric cortical couplings were evaluated by computing EEG coherence between scalp electrode pairs (Gerloff et al., 1998). Significant coherence between EEG electrodes overlying two brain regions has previously been interpreted as evidence of functional coupling (Thatcher, Krause, \& Hrybyk, 1986), mutual information exchange (Rappelsberger \& Petsche, 1988), functional coordination (Gevins et al., 1989), and integrity of connection pathways (Locatelli, Cursi, Liberati, Franceschi, \& Comi, 1998). EEG coherence analysis has also proved to be effective in the modeling of abnormal cortico-cortical connectivity in resting patients with AD (Cutler et al., 1985; Jelic et al., 1996; Leocani \& Comi, 1999; Locatelli et al., 1998). This loss of brain electrical synchronization in $\mathrm{AD}$ has been hypothesized to reflect deafferentation (disconnection mode) of long cortico-cortical fiber tracts from their subcortical cholinergic afferents (Holschneider, Waite, Leuchter, Walton, \& Scremin, 1999).

The working hypothesis of the present study was that in early $\mathrm{AD}$, impaired frontoparietal and interhemispheric neural networks 
(i.e., disconnection mode) controlling voluntary movements would result in negligible variations of corresponding EEG coherence across the baseline, movement, and postmovement periods. This hypothesis would be in line with previous studies showing a reduced EEG coherence in resting patients with $\mathrm{AD}$ compared with control subjects (Comi \& Leocani, 1999; Cutler et al., 1985; Jelic et al., 1996; Locatelli et al., 1998). As an alternative hypothesis, a more effective involvement of these networks would induce enhanced event-related variations of EEG coherence (i.e., cooperation mode).

\section{Method}

\section{Subjects}

Patients with $\mathrm{AD}$ were selected by experienced neurologists from outpatients of Sacro Cuore-Fatebenefratelli (Brescia, Italy), a research institute devoted entirely to diagnosis, treatment, and rehabilitation of patients with AD. The study was approved by the local institutional ethics committee. All experiments were undertaken with the understanding and written consent of each subject or caregiver in accordance with the Code of Ethics of the World Medical Association (Declaration of Helsinki) and the standards established by the Institutional Review Board of Istituto di Ricovero e Cura a Carattere Scientifico "S. Giovanni di Dio Fatebenefratelli," Brescia, Italy.

The group with $\mathrm{AD}$ consisted of 10 right-handed subjects ( 7 women and 3 men) with a mean age of 79.4 years $(S D=8.6$ years $)$. Diagnosis of $\mathrm{AD}$ was made according to criteria of the National Institute of Neurological and Communicative Disorders and Stroke and Alzheimer's Disease and Related Disorders Association (McKhann et al., 1984) and the Diagnostic and Statistical Manual of Mental Disorders (4th ed.; American Psychiatric Association, 1994). All patients underwent general medical, neurological, and psychiatric assessments. Patients were rated with a series of standardized diagnostic and severity instruments including the Mini Mental State Examination (MMSE; Folstein, Folstein, \& McHugh, 1975), the Clinical Dementia Rating Scale (Hughes, Berg, Danziger, Cohen, \& Martin, 1982), the Geriatric Depression Scale (GDS; Yesavage et al., 1982), the Hachinski Ischemic Scale (Rosen, Terry, Fuld, Katzman, \& Peck, 1980), and the Instrumental Activities of Daily Living (Lawton \& Brodie, 1969). In addition, patients underwent diagnostic neuroimaging procedures (head computed tomography or MRI) and laboratory testing to rule out other causes of dementia. Patients were excluded from the study if there was clinical evidence of reversible dementias, such as the pseudodementia of depression (all patients had a GDS score $<14$ ) or the presence of concomitant extrapyramidal signs on exam. In particular, patients with strong fluctuations in standard cognitive performance (Barber et al., 2000; Briel et al., 1999; McKeith et al., 1996), suggesting a possible Lewy body dementia or patients showing features of mixed dementia such as $\mathrm{AD}$ and vascular disease were excluded from the study. The Hachinski score was less than 4. Antidepressant and/or anxyolitic medications were held 24-48 hr prior to EEG recordings. The mean MMSE (corrected for education) and Clinical Deterioration Rate scores were $21.1(S D=4.7)$ and $0.9(S D=$ 0.2 ), respectively.

The control group consisted of 10 right-handed healthy subjects (5 women and 5 men) with a mean age of 72.1 years $(S D=9.1$ years $)$. All healthy controls underwent physical and neurological examinations as well as cognitive screening (including the MMSE and the GDS). Subjects with present or previous history of neurological or psychiatric disease or chronic systemic illnesses (e.g., diabetes mellitus or cardiac, hepatic, or renal insufficiency) and all subjects receiving psychoactive drugs were excluded from the study. The subjects' GDS score had to be lower than 14 in order to exclude potentially depressed individuals.
As expected, women were overrepresented in the $\mathrm{AD}$ group. As there is no previous evidence demonstrating gender-specific effects on EEG rhythms, we felt that this did not interfere with our results.

\section{EEG Recordings}

All details of the experimental task and EEG methods can be found in the companion article by C. Babiloni et al. (2000). The subject was seated in a comfortable reclining armchair placed in a dimly lit, sound-damped, and electrically shielded room. The motor task consisted of brisk right middle-finger extensions triggered by a verbal instruction (i.e., "go") from the experimenter (8-12-s intermovement intervals). Subjects were asked to avoid eye movements during the motor task and for a few seconds postmovement. A brief training session established approximately stable levels of the motor performance. Nineteen scalp electrodes were positioned according to the international 10-20 system (Fp1, Fp2, F7, F3, Fz, F4, F8, T3, C3, Cz, C4, T4, T5, P3, Pz, P4, T6, O1, O2). The EEG data were recorded with $0.3-70.0-\mathrm{Hz}$ bandpass and linked-earlobes reference. To monitor eye movements and mirror finger movements, electrooculogram $(0.3-70.0-\mathrm{Hz}$ bandpass $)$ and surface rectified electromyographic activity of bilateral extensor digitorum muscles $(1-70-\mathrm{Hz}$ bandpass) were also collected. All data were gathered at $256-\mathrm{Hz}$ sampling rate. We obtained about 100 EEG single trials from $4 \mathrm{~s}$ before to $4 \mathrm{~s}$ after the onset of electromyographic response (zero time) for each subject.

\section{Surface Laplacian and Common Average}

The human scalp EEG is recorded against a specific reference and is blurred by head volume conduction, two factors that can artificially inflate the EEG coherence (Andrew \& Pfurtscheller, 1996b; Mima, Matsuoka, \& Hallett, 2000; Nunez, 1995). To minimize these factors, artifact-free (i.e., blinking, eye movements, and mirror movements; number of trials: $49 \pm$ $4[M \pm S E])$ EEG data were transformed by a spline-surface Laplacian estimation (F. Babiloni et al., 1995; F. Babiloni, Carducci, Babiloni, \& Urbano, 1998). This estimation acts as a spatial filter that enhances the activity of EEG cortical sources and protects the data from the effects of the electric reference (Nunez, 1989a, 1989b, 1995). The single trial analysis was then carefully repeated on the Laplacian-transformed EEG data in order to discard those contaminated by computational artifacts.

To control the possible effects of the Laplacian estimation, the coherence analysis was also performed on the artifact-free EEG single trials that had not undergone that estimation. To remove the effects of the electric reference, these trials were re-referenced by the common average reference. The common average procedure includes the averaging of amplitude values at all electrodes and the subtraction of the mean value from the amplitude values at each single electrode.

\section{Between-Electrodes Coherence Analysis}

EEG coherence is a normalized measure of the coupling between two EEG signals at any given frequency (Comi \& Leocani, 1999; Pfurtscheller \& Andrew, 1999; Rappelsberger \& Petsche, 1988). The coherence values were calculated for each frequency bin by Equation 1:

$$
\operatorname{Coh}_{x y}(\lambda)=\left|R_{x y}(\lambda)\right|^{2}=\frac{\left|f_{x y}(\lambda)\right|^{2}}{f_{x x}(\lambda) f_{y y}(\lambda)} .
$$

Equation 1 is the extension of the Pearson's correlation coefficient to complex number pairs. In this equation, $f$ denotes the spectral estimate of two EEG signals $x$ and $y$ for a given frequency bin $(\lambda)$. The numerator contains the cross-spectrum for $x$ and $y\left(f_{x y}\right)$, whereas the denominator contains the respective autospectra for $x\left(f_{x x}\right)$ and $y\left(f_{y y}\right)$. For each frequency bin $(\lambda)$, the coherence value $\left(C o h_{x y}\right)$ is obtained by squaring the magnitude of the complex correlation coefficient $R$. This procedure returns a real number between 0 (no coherence) and 1 ( $\max$ coherence). 
The between-electrodes EEG coherence was computed only from the internal electrodes used in the 10-20 system montage (F3, Fz, F4, C3, Cz, C4, P3, Pz, P4). At these electrodes, the spline-surface Laplacian estimate is considered to be reliable (Perrin, Pernier, Bertrand, \& Echallier, 1989). In particular, the between-electrodes EEG coherence was computed from F3-P3, Fz-Pz, and F4-P4 for the evaluation of functional frontoparietal connectivity as well as from $\mathrm{F} 3-\mathrm{F} 4, \mathrm{C} 3-\mathrm{C} 4$, and $\mathrm{P} 3-\mathrm{P} 4$ for the evaluation of functional interhemispheric connectivity. The between-electrodes EEG coherence was calculated at baseline or rest (from $-4 \mathrm{~s}$ to $-3 \mathrm{~s}$ ), movement (from $0 \mathrm{~s}$ to $1 \mathrm{~s}$ ), and postmovement (from $1 \mathrm{~s}$ to $2 \mathrm{~s}$ ) periods.

Broadband EEG coherence was calculated for alpha $(9-11 \mathrm{~Hz})$, beta 1 $(14-16 \mathrm{~Hz})$, and beta $2(20-22 \mathrm{~Hz})$ frequency bands. These bands were selected for two reasons. First, they are considered particularly sensitive to movement-related EEG coherence in humans (Gerloff et al., 1998). Second, visual inspection of the individual coherence spectra confirmed the presence of EEG coherence peaks at those frequency bands (see Figure 2). Of note, no clear EEG coherence peak was observed in the gamma band for a simple motor task. Therefore, the gamma band was not considered in the data analysis of the present study.

The statistical analysis of the coherence strictly followed the procedure reported by Halliday et al. (1995). According to this procedure, the threshold level of the statistically significant $(95 \%)$ EEG coherence was computed on the basis of the number of EEG single trials. Given the mean number of EEG single trials across subjects $(49 \pm 4 S E)$, the statistical threshold for the coherence at $p<.05$ was lower than .1 (i.e., .06).

\section{Statistical Evaluation}

Statistical analysis of the EEG coherence (independent variable) was performed with two analysis of variance (ANOVA) designs. The subject's age was used as a covariate. The Mauchley's test served to evaluate the sphericity assumption for both ANOVAs. We used the Greenhouse-
Geisser procedure to correct the degrees of freedom and the Duncan test for post hoc comparisons $(p<.05)$.

The first ANOVA design included the variables subject (control, AD), band (alpha, beta 1, beta 2), electrode pair (F4-P4, Fz-Pz, F3-P3), and period (rest, movement, postmovement) to test the functional frontoparietal connectivity. The second ANOVA had the same design and tested the functional connectivity between the two hemispheres (as a unique difference, electrode pair: F3-F4, C3-C4, P3-P4).

With reference to the working hypotheses, the disconnection mode of the cortical coupling predicted a statistical ANOVA interaction including Subject (control, AD) $\times$ Period (rest, movement, postmovement) as a sign of unreactive EEG coherence in patients with $\mathrm{AD}$ across rest, movement, postmovement periods. In contrast, the cooperation mode of the cortical coupling predicted significant EEG coherence changes in AD patients across rest, movement, and postmovement periods.

\section{Results}

Figure 2 plots the spectra of the EEG coherence computed across subjects from the Laplacian-transformed EEG data at the baseline (rest), movement, and postmovement periods (lasting $1 \mathrm{~s}$ each). These spectra showed an evident alpha peak in the frontal and parietal connections.

The ANOVA of frontoparietal EEG coherence showed a significant Subject (control, AD) $\times$ Period (rest, movement, postmovement) interaction, $F(2,18)=5.72, M S E=0.0039, p<.01$. The group means $( \pm S E$ ) of the EEG coherence ANOVA interaction are shown in Figure 3. Note that no significant ANOVA interaction including the variable band was observed, thus indicating that there were no statistical differences in the EEG coherence values
NOLD
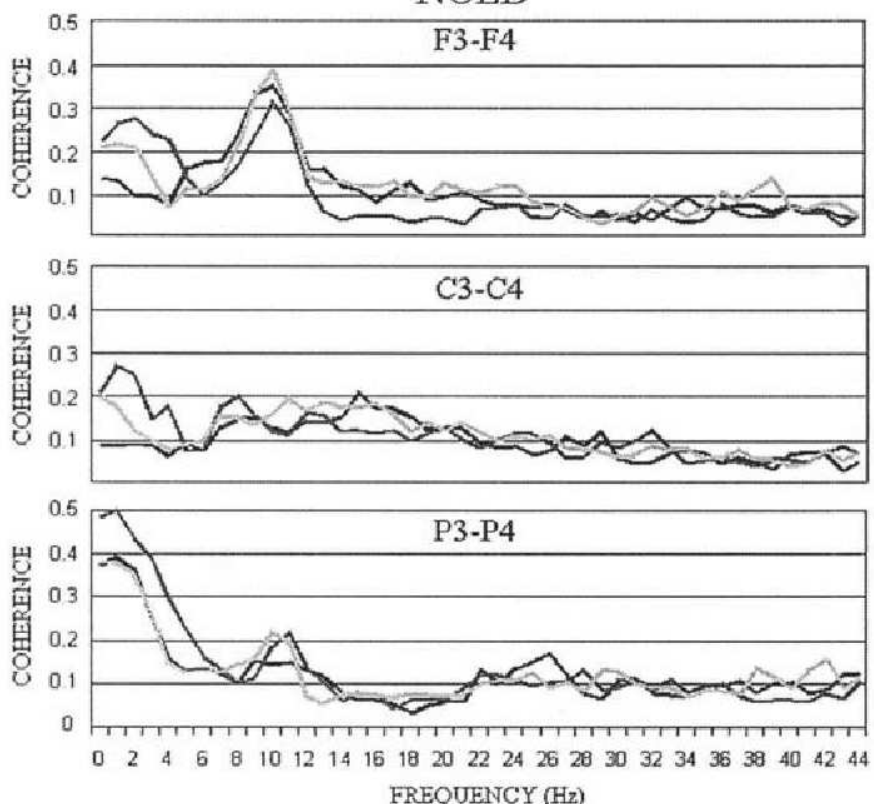

FREQUENCY $(\mathrm{Hz})$
$\mathrm{AD}$
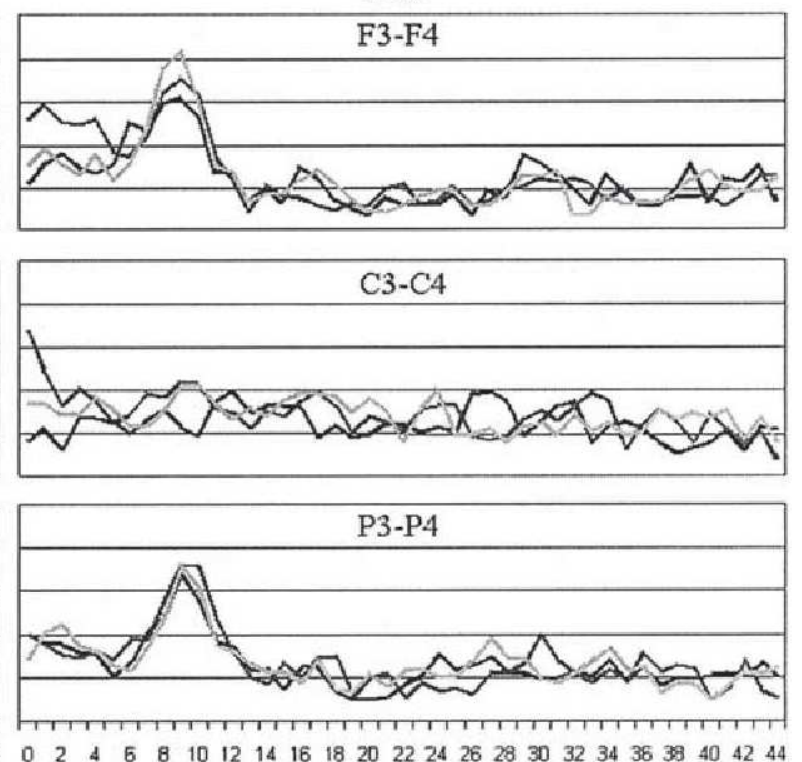

FREQUENCY (Hz)

- REST - MOV - POST-MOV

Figure 2. Across-subject electroencephalographic (EEG) coherence spectra computed from the Laplaciantransformed EEG data at the baseline (REST), movement (MOV), and postmovement (POST-MOV) periods, lasting $1 \mathrm{~s}$ each. $\mathrm{AD}=$ Alzheimer's disease; NOLD $=$ control subjects. F3 and F4, C3 and C4, and P3 and P4 refer to frontal, central, and parietal electrode sites, respectively. 


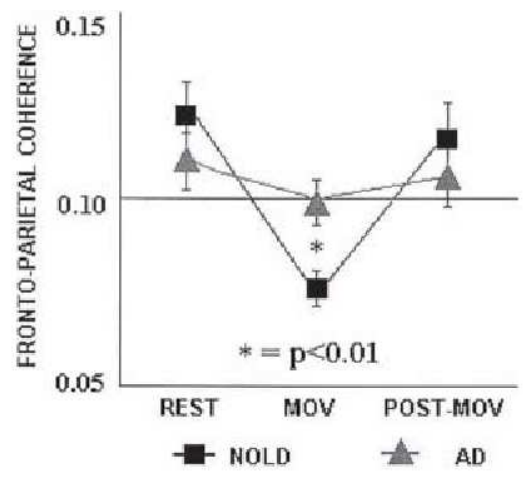

Figure 3. Across-subject means ( $\pm S E M$ ) of frontoparietal electroencephalographic coherence as provided by the analysis of variance (ANOVA) design. The ANOVA design included the variables subject (control [NOLD], Alzheimer's disease [AD]), band (alpha, beta 1, beta 2), electrode pair (F4-P4, Fz-Pz, F3-P3), and period (rest, movement [MOV], postmovement [POST-MOV]). In particular, these means refer to a significant Subject (control, AD) $\times$ Period (rest, movement, postmovement) interaction, $F(2,18)=5.72, M S E=0.0039, p<.01$. Duncan post hoc testing results are indicated by an asterisk $(p<.01)$.

at the alpha, beta 1 , and beta 2 bands. Duncan post hoc testing indicated that, regardless of the frequency bands and electrode pairs, a strong variation of the EEG coherence was observed in control subjects but not in patients with $\mathrm{AD}$. In control subjects, there was a reduction of the EEG coherence from the rest to the movement periods $(p<.00005)$ and an increase of the EEG coherence from the movement to the postmovement periods $(p<$ $.0005)$. Furthermore, the frontoparietal EEG coherence was lower in control subjects than in patients with $\mathrm{AD}$ during the movement period $(p<.01)$

The ANOVA of the interhemispheric EEG coherence also pointed to a significant Subject (control, AD) $\times$ Period (rest, movement, postmovement) interaction $F(2,18)=4.03, M S E=$ $0.0044, p<.03$. Figure 4 plots the group means $( \pm S E)$ of the EEG coherence for this interaction. Again, no significant ANOVA interaction including the variable band was observed. Duncan post hoc testing substantiated a strong variation of the EEG coherence in control subjects, regardless of the EEG bands and electrode pairs. As for the frontoparietal EEG coherence, there was a reduction of the EEG coherence from the rest to the movement periods $(p<.03)$ and an increase of the EEG coherence from the movement to the postmovement periods $(p<.02)$. In contrast, no significant variation of the EEG coherence was seen in patients with $\mathrm{AD}$. It is noteworthy that the interhemisphere EEG coherence was stronger in control subjects than in patients with $\mathrm{AD}$ during the rest $(p<.002)$ and the postmovement $(p<.009)$ periods.

To demonstrate the validity of the above results, we performed two control analyses. The first control analysis addressed the issue of the spatial specificity of the results on long-range frontoparietal connectivity. We used two ANOVA designs for the EEG coherence computed from short-range connectivity such as frontocentral (F3-C3, Fz-Cz, F4-C4) and centroparietal (C3-P3, Cz-Pz, C4$\mathrm{P} 4)$. In contrast to the previous ANOVA relative to long-range frontoparietal connectivity, no significant Subject (control, AD) $\times$ Period (rest, movement, postmovement) interaction was found $(p<.3)$.
The second control analysis addressed the validity of the coherence reduction in control subjects during the movement period compared with the rest and postmovement periods. For this purpose, the coherence was computed from the common averaged EEG data. The results statistically confirmed the aforementioned coherence reduction and the significant Subject $\times$ Period interactions $(p<.03)$.

\section{Discussion}

Surface Laplacian estimates should always be interpreted with caution. First, surface Laplacian maxima cannot always overlie cortical sources of EEG potentials because of the influence of both radial and tangential cortical generators (F. Babiloni et al., 1996). However, this limitation might be less important here because radial cortical sources are supposed to prevail in the generation of EEG rhythmicity. Second, surface Laplacian estimates might not be fully reliable when computed at the border electrodes. For this reason, temporal electrodes were not considered here (Nunez, 1995). Third, the low resolution of our EEG spatial sampling (19 recording channels) permitted the evaluation of only large regions of interest such as frontal, central, and parietal areas of both hemispheres. Such a coarse level of anatomical description took into account the slight changes in brain volume provoked by atrophy processes occurring at early $\mathrm{AD}$ stages.

Another important methodological issue is the influence of spline Laplacian estimators on EEG coherence. Previous studies have reported the risk of artifactual EEG coherence computed from spline Laplacian-transformed data, in the sense of an inflated (Biggins, Fein, Raz, \& Amir, 1991) or reduced (Nunez, 1995; Rappelsberger, 1989) EEG coherence. However, more recent investigations on simulated and real EEG data have shown that artifactual effects are negligible when the spline Laplacian functions are correctly implemented and used (Nunez et al., 1997; Pascual-Marqui, 1993; Perrin, 1992). Furthermore, computational artifacts from Laplacian estimates are very small when EEG co-

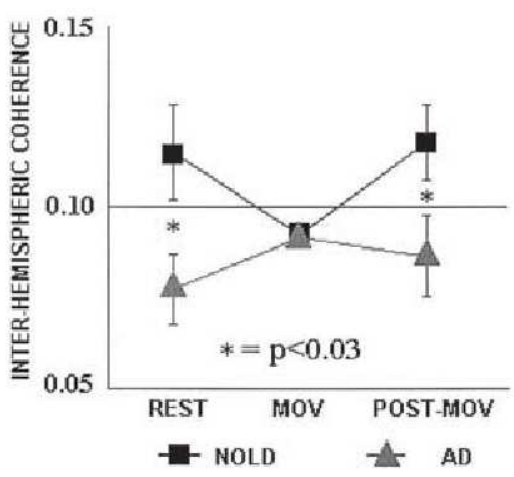

Figure 4. Across-subject means ( $\pm S E M$ ) of interhemispheric electroencephalographic coherence as provided by the analysis of variance (ANOVA) design. The ANOVA design included the variables subject (control [NOLD], Alzheimer's disease [AD]), band (alpha, beta 1, beta 2), electrode pair (F3-F4, C3-C4, P3-P4), and period (rest, movement [MOV], postmovement [POST-MOV]). In particular, these means refer to a significant Subject (control, AD) $\times$ Period (rest, movement, postmovement) interaction, $F(2,18)=4.03, M S E=0.0044, p<.03$. Duncan post hoc testing results are indicated by an asterisk $(p<.03)$. 
herence is calculated at scalp electrodes more distant than 3 or 4 $\mathrm{cm}$ and when border electrodes are not considered (Nunez et al., 1997, 1999). In this regard, our spline Laplacian functions have been proved to be correctly implemented on simulated and real EEG data (C. Babiloni et al., 1999, 2000, 2001, 2002; F. Babiloni et al., 1995, 1996). Furthermore, we strictly followed the aforementioned guidelines for the use of surface Laplacian estimation for EEG coherence analysis.

\section{Movement-Related EEG Coherence in Control Subjects}

As expected, the interhemispheric and frontoparietal EEG coherence changed significantly from the baseline to postmovement periods in control subjects. Compared with the baseline and postmovement periods, the coherence decreased during the movement period. This result was not due to the surface Laplacian estimation given that similar findings were obtained by computing the coherence from common averaged EEG data. From a physiological point of view, the movement-related reduction of the coherence can be explained by the brain wave theory (Nunez, 1995, pp. 694-698 of the Appendix). During the rest period, a global synchronization mechanism made the idling rhythms of distant frontoparietal and interhemispheric cortical areas more coherent. During the movement, a local synchronization mechanism induced a task-specific and transient (time-varying) modulation of the cortical oscillators. As a result, a decrement (rather than an increment) of the functional coupling occurred among oscillators sensitive to the global rather than the local mechanisms. These mechanisms used diffuse (global) or circumscribed (local) thalamocortical and cortico-cortical connections (Andrew \& Pfurtscheller, 1996a, 1996b; Gevins et al., 1989, 1990; Holschneider et al., 1999; Nunez, 1995; Pfurtscheller \& Lopes da Silva, 1999; Thatcher et al., 1986).

Our finding of a reduced interhemispheric EEG coherence accompanying the movement merits a detailed discussion because of the controversy surrounding previous evidence on this matter. On the one hand, a complex interhemispheric pattern of increased (Andres et al., 1999; Gerloff et al., 1998), sustained, or decreased (Leocani, Toro, Manganotti, Zhuang, \& Hallett, 1997) EEG coherence during the movement execution has been previously reported. On the other hand, movement-related EEG coherence has been found to decrease between sensorimotor cortices when computed from reference-free Laplacian-transformed EEG data (Andrew \& Pfurtscheller 1996a, 1996b, 1999; Florian, Andrew, \& Pfurtscheller, 1998). During the movement, these oscillations would reflect an upper alpha rhythm sensitive to the event and having no interhemispheric coherence (i.e., mu rhythm, 10-12 $\mathrm{Hz}$ ). This upper alpha rhythm would be embedded with a local lower alpha rhythm having constant interhemispheric coherence $(8-10 \mathrm{~Hz})$. This explanation accounts for the previous finding showing that the two sensorimotor areas generated independent EEG rhythms (van Leeuwen, Wieneke, Spoelstra, \& Versteeg, 1978) and that the interhemispheric EEG coherence during the movement was independent of the volume conduction of posterior alpha rhythm (Mima et al., 2000).

The present finding of a decreased coherence during the movement is apparently at odds with a recent electrocorticographic study (Ohara et al., 2001) showing an increase of the coherence over contralateral premotor and motor areas during the movement.
First of all, the study revealed the increased coherence in the contralateral motor areas but did not test the coherence between the two hemispheres. Furthermore, it should be stressed that electrocorticographic and scalp EEG recordings probe neural populations at very different spatial scales. According to Nunez's (1995) theory, electrocorticographic electrodes are very close to each other and probe local synchronization mechanisms. On the contrary, the scalp EEG electrodes are distant and probe long-range coupling or decoupling of neural populations sensitive to the global rather than the local mechanisms.

\section{Movement-Related EEG Coherence in Patients With AD}

In contrast to control subjects and despite a strong ERD/ERS (C. Babiloni et al., 2000), patients with AD showed no change in the frontoparietal and interhemispheric EEG coherence across the baseline, movement, and postmovement periods as well as a general low coherence during the inactive (baseline and postmovement) periods. It is possible that these results were due to an endurance entrainment of the task-specific local synchronization mechanism that decoupled the EEG rhythms at distant cortical regions. The above speculation predicts in early $\mathrm{AD}$ a sustained excitation or disinhibition and inefficient information transfer across interhemispheric fiber tracts during inactive periods. This prediction is in line with recent findings showing an increased sensorimotor cortical excitability in $\mathrm{AD}$ as revealed by somatosensory evoked potentials (Ferri et al., 1996) and transcranial magnetic stimulation (Alagona et al., 2001; Ferreri et al., 2003; Pennisi et al., 2002). These findings raise the issue of the role of inhibitory neurotransmitters in mild AD.

The present results extend, to the inactive (baseline and postmovement) periods of a motor task, previous evidence showing that interhemispheric EEG coherence values are lower in resting AD patients than in control subjects (Locatelli et al., 1998; Wada, Nanbu, Kikuchi, et al., 1998; Wada, Nanbu, Koshino, et al., 1998). According to the brain wave theory (Nunez, 1995, pp. 694-698 of the Appendix), there would be a partial failure of a global synchronization mechanism in $\mathrm{AD}$ associated with a background sensorimotor hyperexcitability. This explanation is compatible with increased motor-evoked potentials previously induced by transcranial magnetic stimulations in resting patients with $\mathrm{AD}$ as compared with control subjects (Alagona et al., 2001; Ferreri et al., 2003; Pennisi et al., 2002). In contrast, there is evidence indicating that the interhemispheric EEG coherence at rest is mainly due to the integrity of cortico-cortical fibers, such as the anterior commisure and the corpus callosum, linking the two hemispheres (Knyazeva \& Innocenti, 2001). For this reason, the abnormal interhemispheric EEG coherence in $\mathrm{AD}$ has been previously ascribed to an impairment of these fibers (Knott, Mohr, Mahoney, \& Ilivitsky, 2000; Locatelli et al., 1998; Stevens et al., 2001; Wada, Nanbu, Kikuchi, et al., 1998; Wada, Nanbu, Koshino, et al., 1998). As an empirical confirmation, MRI studies have demonstrated a significant reduction in thickness of corpus callosum in patients with AD (Janowsky, Kaye, \& Carper, 1996; Vermersch, Scheltens, Barkhof, Steinling, \& Leys, 1994).

Abnormal coherence of frontoparietal EEG rhythms has been repeatedly observed in resting AD patients (Besthorn et al., 1994; Comi \& Leocani, 1999; Dunkin, Leuchter, Newton, \& Cook, 1994; Le Roc'h, Rancurel, Piotrenaud, Bourgin, \& Sebban, 1993; 
Leuchter et al., 1992; Leuchter, Spar, Walter, \& Weiner, 1987; Locatelli et al., 1998) in association with a decrease of regional cerebral blood flow (Passero, Rocchi, Vatti, Burgalassi, \& Battistini, 1995; Sloan, Fenton, Kennedy, \& MacLennan, 1994). These EEG coherence findings have been interpreted as being due mainly to an impairment of the long cholinergic pathways connecting temporal, parietal, and occipital areas to the frontal areas (Holschneider et al., 1999; Locatelli et al., 1998). Indeed, associative cortical areas of patients with $\mathrm{AD}$ lose afferent and efferent connections in parallel with the damage and death of pyramidal neurons, for which long axon connections and synapses would be the anatomical basis of the functional frontoparietal coupling (Pearson, Esiri, Hiorns, Wilcock, \& Powell, 1985). However, it is possible that the abnormal frontoparietal EEG coherence in patients with $\mathrm{AD}$ could be provoked in part by a concomitant deficit in the thalamocortical gating of information flows from the motor (basal ganglia and cerebellum) and sensory (skin, joint, and muscle receptors) thalamic nuclei to the cortex. Of note, the functional connectivity as revealed here by the coherence results would not depend on the magnitude of the brain alpha rhythm at rest, which was lower in patients with mild $\mathrm{AD}$ than in control subjects. In fact, the coherence estimation is independent of the magnitude of the spectral power density.

As a final consideration, the results of the present study extend previous evidence (Neubauer, Fink, \& Schrausser, 2002) concerning the neural efficiency as shown by the comparison between patients with $\mathrm{AD}$ and healthy controls in that the greater postmovement beta ERS in patients with $\mathrm{AD}$ than inhealthy controls was associated with neither better motor performance as revealed by the reaction time (C. Babiloni et al., 2000) nor coordination between the two hemispheres from the preparation to the execution of the movement.

\section{Conclusion}

The present study indicates that movement-related EEG coherence is altered in early $\mathrm{AD}$ and that it reflects an impairment of the frontoparietal and interhemispheric cortical coupling. In general, the patients with $\mathrm{AD}$ showed an unreactive frontoparietal and interhemispheric EEG coherence across the baseline, movement, and postmovement periods. Furthermore, they presented a low EEG coherence at the inactive periods (baseline and postmovement). These results suggest that the strong movement-related ERD/ERS previously observed in patients with early $\mathrm{AD}$ over the frontal and ipsilateral central sensorimotor areas (C. Babiloni et al., 2000 ) would be well accounted for by an impaired cortical coupling within the networks involved in the motor control (i.e., the present disconnection mode hypothesis). According to the aforementioned brain wave theory (Nunez, 1995), such a disconnection mode could be due to an altered entrainment of the task-specific local synchronization mechanism in the framework of the inputs provided by the global synchronization mechanism. Further investigations should evaluate the clinical utility of these findings in comparison with the standard quantitative EEG analysis of resting subjects (Fenton, 1986; Ihl, Dierks, Martin, Froolich, \& Maurer, 1996; Leuchter et al., 1993; Rodriguez et al., 1998; Rodriguez, Copello, Vitali, Perego, \& Nobili, 1999).

\section{References}

Alagona, G., Bella, R., Ferri, R., Carnemolla, A., Pappalardo, A., Costanzo, E., \& Pennisi, G. (2001, November 13). Transcranial magnetic stimulation in Alzheimer disease: Motor cortex excitability and cognitive severity. Neuroscience Letters, 314(1-2), 57-60.

American Psychiatric Association. (1994). Diagnostic and statistical manual of mental disorders (4th ed.). Washington, DC: Author.

Andres, F. G., Mima, T., Schulman, A. E., Dichgans, J., Hallett, M., \& Gerloff, C. (1999). Functional coupling of human cortical sensorimotor areas during bimanual skill acquisition. Brain, 122, 855-870.

Andrew, C., \& Pfurtscheller, G. (1996a). Dependence of coherence measurements on EEG derivation type. Medical \& Biological Engineering \& Computing, 34, 232-238.

Andrew, C., \& Pfurtscheller, G. (1996b). Event-related coherence as a tool for studying dynamic interaction of brain regions. Electroencephalography and Clinical Neurophysiology, 98, 144-148.

Andrew, C., \& Pfurtscheller, G. (1999, October 1). Lack of bilateral coherence of post-movement central beta oscillations in the human electroencephalogram. Neuroscience Letters, 273(2), 89-92.

Babiloni, C., Babiloni, F., Carducci, F., Cincotti, F., Del Percio, C., De Pino, G., et al. (2000). Movement-related electroencephalographic reactivity in Alzheimer disease. Neurolmage, 12, 139-146.

Babiloni, C., Babiloni, F., Carducci, F., Cincotti, F., Rosciarelli, F., ArendtNielsen, L., et al. (2002). Human brain oscillatory activity phase-locked to painful electrical stimulations: A multi-channel EEG study. Human Brain Mapping, 15, 112-123.

Babiloni, C., Babiloni, F., Carducci, F., Cincotti, F., Rosciarelli, F., Rossini, P., et al. (2001). Mapping of early and late human somatosensory evoked brain potentials to phasic galvanic painful stimulation. Human Brain Mapping, 12, 168-179.

Babiloni, C., Carducci, F., Cincotti, F., Rossini, P. M., Neuper, C., Pfurtscheller, G., \& Babiloni, F. (1999). Human movement-related potentials vs desynchronization of EEG alpha rhythm: A high-resolution EEG study. Neurolmage, 10, 658-665.

Babiloni, F., Babiloni, C., Carducci, F., Fattorini, L., Onorati, P., \& Urbano, A. (1995). Performances of surface Laplacian estimators: A study of simulated and real scalp potential distributions. Brain Topography, 8, 33-45.

Babiloni, F., Babiloni, C., Carducci, F., Fattorini, L., Onorati, P., \& Urbano, A. (1996). Spline Laplacian estimate of EEG potentials over a realistic magnetic resonance-constructed scalp surface model. Electroencephalography and Clinical Neurophysiology, 98, 363-373.

Babiloni, F., Carducci, F., Babiloni, C., \& Urbano, A. (1998). Improved realistic Laplacian estimate of highly-sampled EEG potentials with regularization techniques. Electroencephalography and Clinical Neurophysiology, 106, 336-343.

Barber, R., Gholkar, A., Scheltens, P., Ballard, C., McKeith, I. G., \& O'Brien, J. T. (2000). MRI volumetric correlates of white matter lesions in dementia with Lewy bodies and Alzheimer's disease. International Journal of Geriatric Psychiatry, 15, 911-916.

Besthorn, C., Forstl, H., Geiger-Kabish, C., Sattel, H., Gasser, T., \& Schreiter-Gasser, U. (1994). EEG coherence in Alzheimer disease. Electroencephalography and Clinical Neurophysiology, 90, 242-245.

Biggins, C. A., Fein, G., Raz, J., \& Amir, A. (1991). Artifactually high coherences result from using spherical spline computation of scalp current density. Electroencephalography and Clinical Neurophysiology, 79, 413-419.

Briel, R. C., McKeith, I. G., Barker, W. A., Hewitt, Y., Perry, R. H., Ince, P. G., \& Fairbairn, A. F. (1999). EEG findings in dementia with Lewy bodies and Alzheimer's disease. Journal of Neurology, Neurosurgery, and Psychiatry, 66, 401-403.

Comi, G., \& Leocani, L. (1999). Neurophysiological imaging techniques in dementia. Italian Journal of Neurological Sciences, 20 (Suppl. 5), S265S269. 
Cutler, N. R., Haxby, J. V., Duara, R., Grady, C. L., Moore, A. M., Parisi, J. E., et al. (1985). Brain metabolism as measured with positron emission tomography: Serial assessment in a patient with familial Alzheimer's disease. Neurology, 35, 1556-1561.

Dunkin, J. J., Leuchter, A. F., Newton, T. F., \& Cook, I. A. (1994). Reduced EEG coherence in dementia: State or trait marker? Biological Psychiatry, 35, 870-879.

Fenton, G. W. (1986). Electrophysiology of Alzheimer's disease. British Medical Bulletin, 42, 29-33.

Ferreri, F., Pauri, F., Pasqualetti, P., Fini, R., Dal Forno, G., \& Rossini, P. M. (2003). Motor cortex excitability in Alzheimer's disease: A transcranial magnetic stimulation study. Annals of Neurology, 53, 102 108.

Ferri, R., Del Gracco, S., Elia, M., Musumeci, S. A., Spada, R., \& Stefanini, M. C. (1996). Scalp topographic mapping of middle-latency somatosensory evoked potentials in normal aging and dementia. Neurophysiologie Clinique, 26, 311-319.

Florian, G., Andrew, C., \& Pfurtscheller, G. (1998). Do changes in coherence always reflect changes in functional coupling? Electroencephalography and Clinical Neurophysiology, 106, 87-91.

Folstein, M. F., Folstein, S. E., \& McHugh, P. R. (1975). "Mini Mental State": A practical method for grading the cognitive state of patients for the clinician. Journal of Psychiatric Research, 12, 189-198.

Gerloff, C., Richard, J., Hadley, J., Schulman, A. E., Honda, M., \& Hallett, M. (1998). Functional coupling and regional activation of human cortical motor areas during simple, internally paced and externally paced finger movements. Brain, 121, 1513-1531.

Gevins, A. S., Bressler, S. L., Cutillo, B. A., Illes, J., Miller, J. C., Stern, J., \& Jex, H. R. (1990). Effects of prolonged mental work on functional brain topography. Electroencephalography and Clinical Neurophysiology, 76, 339-350.

Gevins, A. S., Cutillo, B. A., Bressler, S. L., Morgan, N. H., White, R. M., Illes, J., \& Greer, D. S. (1989). Event-related covariances during a bimanual visuomotor task. II. Preparation and feedback. Electroencephalography and Clinical Neurophysiology, 74, 147-160.

Goldman, W. P., Baty, J. D., Buckles, V. D., Sahrmann, S., \& Morris, J. C. (1999). Motor dysfunction in mildly demented AD individuals without extrapyramidal signs. Neurology, 53, 956-962.

Halliday, D. M., Rosenberg, J. R., Amjad, A. M., Breeze, P., Conway, B. A., \& Farmer, S. F. (1995). A framework for the analysis of mixed time series/point process data - Theory and application to the study of physiological tremor, single motor unit discharges and electromyograms. Progress in Biophysics and Molecular Biology, 64, 237-278.

Holschneider, D. P., Waite, J. J., Leuchter, A. F., Walton, N. Y., \& Scremin, O. U. (1999). Changes in electrocortical power and coherence in response to the selective cholinergic immunotoxin 192 IgG-saporin. Experimental Brain Research, 126, 270-280.

Hughes, C. P., Berg, L., Danziger, W. L., Cohen, L. A., \& Martin, R. L. (1982). A new clinical rating scale for the staging of dementia. British Journal of Psychiatry, 140, 1225-1230.

Ihl, R., Dierks, T., Martin, E. M., Froolich, L., \& Maurer, K. (1996). Topography of the maximum of the amplitude of EEG frequency bands in dementia of the Alzheimer type. Biological Psychiatry, 39, 319-325.

Janowsky, J. S., Kaye, J. A., \& Carper, R. A. (1996). Atrophy of the corpus callosum in Alzheimer's disease versus healthy aging. Journal of the American Geriatrics Society, 44, 798-803.

Jelic, V., Shigeta, M., Julin, P., Almkvist, O., Winblad, B., \& Wahlund, L. O. (1996). Quantitative electroencephalography power and coherence in Alzheimer's disease and mild cognitive impairment. Dementia, 7, 314-323.

Knott, V., Mohr, E., Mahoney, C., \& Ilivitsky, V. (2000). Electroencephalographic coherence in Alzheimer's disease: Comparisons with a control group and population norms. Journal of Geriatric Psychiatry and Neurology, 13, 1-8.
Knyazeva, M. G., \& Innocenti, G. M. (2001). EEG coherence studies in the normal brain and after early-onset cortical pathologies. Brain Research. Brain Research Reviews, 36, 119-128.

Lawton, M. P., \& Brodie, E. M. (1969). Assessment of older people: Self maintaining and instrumental activity of daily living. Journal of Gerontology, 9, 179-186.

Leocani, L., \& Comi, G. (1999). EEG coherence in pathological conditions. Journal of Clinical Neurophysiology, 16, 548-555.

Leocani, L., Toro, C., Manganotti, P., Zhuang, P., \& Hallett, M. (1997). Event-related coherence and event-related desynchronization/synchronization in the $10 \mathrm{~Hz}$ and $20 \mathrm{~Hz}$ EEG during self-paced movements. Electroencephalography and Clinical Neurophysiology, 104, 199-206.

Le Roc'h, K., Rancurel, G., Piotrenaud, J., Bourgin, P., \& Sebban, C. (1993). Fluidité verbale et cohérence EEG dans la maladie d'Alzheimer [Verbal fluidity and EEG coherence in Alzheimer's disease]. Neurophysiologie Clinique, 23, 422-433.

Leuchter, A. F., Cook, I. A., Newton, T. F., Dunkin, J., Walter, D. O., Rosenberg-Thompson, S., et al. (1993). Regional differences in brain electrical activity in dementia: Use of spectral power and spectral ratio measures. Electroencephalography and Clinical Neurophysiology, 87, 385-393.

Leuchter, A. F., Newton, T. F., Cook, I. A., Walter, D. O., RosenbergThompson, S., \& Lachenbruch, P. A. (1992). Changes in brain functional connectivity in Alzheimer-type and multi-infarct dementia. Brain, $115,1543-1561$.

Leuchter, A. F., Spar, J. E., Walter, D. O., \& Weiner, H. (1987). Electroencephalographic spectra and coherence in the diagnosis of Alzheimer's-type and multi-infarct dementia. A pilot study. Archives of General Psychiatry, 44, 993-998.

Locatelli, T., Cursi, M., Liberati, D., Franceschi, M., \& Comi, G. (1998). EEG coherence in Alzheimer's disease. Electroencephalography and Clinical Neurophysiology, 106, 229-237.

McKeith, I. G., Galasko, D., Kosaka, K., Perry, E. K., Dickson, D. W., \& Hansen, L. A. (1996). Consensus guidelines for the clinical and pathologic diagnosis of dementia with Lewy bodies (DLB): Report of the Consortium on DLB international workshop. Neurology, 47, 1113-1124.

McKhann, G., Drachman, D., Folstein, M., Katzman, R., Price, D., \& Stadlan, E. M. (1984). Clinical diagnosis of Alzheimer's disease: Report of the NINCDS-ADRDA Work Group under the auspices of Department of Health and Human Services Task Force on Alzheimer's Disease. Neurology, 34, 939-944.

Mima, T., Matsuoka, T., \& Hallett, M. (2000, June 23). Functional coupling of human right and left cortical motor areas demonstrated with partial coherence analysis. Neuroscience Letters, 287(2), 93-96.

Neubauer, A. C., Fink, A., \& Schrausser, D. G. (2002). Intelligence and neural efficiency: The influence of task content and sex on the brain-IQ relationship. Intelligence, 30, 515-536.

Nunez, P. L. (1989a). Estimation of large scale neocortical source activity with EEG surface Laplacians. Brain Topography, 2, 141-154.

Nunez, P. L. (1989b). Generation of human EEG by a combination of long and short range neocortical interactions. Brain Topography, 1, 199-215.

Nunez, P. (1995). Neocortical dynamics and human EEG rhythms. New York: Oxford University Press.

Nunez, P. L., Silberstein, R. B., Shi, Z., Carpenter, M. R., Srinivasan, R., Tucker, D. M., et al. (1999). EEG coherency II: Experimental comparisons of multiple measures. Clinical Neurophysiology, 110, 469-486.

Nunez, P. L., Srinivasan, R., Westdorp, A. F., Wijesinghe, R. S., Tucker, D. M., Silberstein, R. B., \& Cadusch, P. J. (1997). EEG coherency. I: Statistics, reference electrode, volume conduction, Laplacians, cortical imaging, and interpretation at multiple scales. Electroencephalography and Clinical Neurophysiology, 103, 499-515.

Ohara, S., Mima, T., Baba, K., Ikeda, A., Kunieda, T., Matsumoto, R., et al. (2001). Increased synchronization of cortical oscillatory activities 
between human supplementary motor and primary sensorimotor areas during voluntary movement. Journal of Neuroscience, 21, 9377-9386.

Pascual-Marqui, R. D. (1993). The spherical spline Laplacian does not produce artifactually high coherences: Comments on two articles by Biggins et al. Electroencephalography and Clinical Neurophysiology, 87, 62-64.

Passero, S., Rocchi, R., Vatti, G., Burgalassi, L., \& Battistini, N. (1995). Quantitative EEG mapping, regional cerebral blood flow, and neuropsychological function in Alzheimer's disease. Dementia, 6, 148-156.

Pearson, R. C., Esiri, M. M., Hiorns, R. W., Wilcock, G. K., \& Powell, T. P. (1985). Anatomical correlates of the distribution of the pathological changes in the neocortex in Alzheimer disease. Proceedings of the National Academy of Sciences, USA, 82, 4531-4534.

Pennisi, G., Alagona, G., Ferri, R., Greco, S., Santonocito, D., Pappalardo, A., \& Bella, R. (2002, September 6). Motor cortex excitability in Alzheimer disease: One year follow-up study. Neuroscience Letters, $329(3), 293$.

Perrin, F. (1992). Comments on article by Biggins et al. Electroencephalography and Clinical Neurophysiology, 83, 171-174.

Perrin, F., Pernier, J., Bertrand, O., \& Echallier, J. F. (1989). Spherical spline for potential and current density mapping. Electroencephalography and Clinical Neurophysiology, 72, 184-187.

Pfurtscheller, G., \& Andrew, C. (1999). Event-related changes of band power and coherence: Methodology and interpretation. Journal of Clinical Neurophysiology, 16, 512-519.

Pfurtscheller, G., \& Lopes da Silva, F. H. (1999). Event-related EEG/MEG synchronization and desynchronization: Basic principles. Clinical Neurophysiology, 110, 1842-1857.

Pfurtscheller, G., Neuper, C., Andrew, C., \& Edlinger, G. (1997). Foot and hand area mu rhythms. International Journal of Psychophysiology, 26, 121-135.

Pfurtscheller, G., Pichler-Zalaudek, K., \& Neuper, C. (1999). ERD and ERS in voluntary movement of different limbs. In G. Pfurtscheller \& F. H. Lopes da Silva (Eds.), Handbook of electroencephalography and clinical neurophysiology: No. 6. Event-related desynchronization/synchronization (Revised ed., pp. 245-268). Amsterdam: Elsevier.

Rappelsberger, P. (1989). The reference problem and mapping of coherence: A simulation study. Brain Topography, 2, 63-72.

Rappelsberger, P., \& Petsche, H. (1988). Probability mapping: Power and coherence analyses of cognitive processes. Brain Topography, 1, 46-54.

Rodriguez, G., Copello, F., Vitali, P., Perego, G., \& Nobili, F. (1999). EEG spectral profile to stage Alzheimer's disease. Clinical Neurophysiology, 110, 1831-1837.
Rodriguez, G., Nobili, F., Rocca, G., De Carli, F., Gianelli, M. V., \& Rosadini, G. (1998). Quantitative electroencephalography and regional cerebral blood flow: Discriminant analysis between Alzheimer's patients and healthy controls. Dementia and Geriatric Cognitive Disorders, 9, $274-283$.

Rosen, W. G., Terry, R. D., Fuld, P. A., Katzman, R., \& Peck, A. (1980). Pathological verification of ischemic score in differentiation of dementias. Annals of Neurology, 7, 486-488.

Sloan, E. P., Fenton, G. W., Kennedy, N. S., \& MacLennan, J. M. (1994). Neurophysiology and SPECT cerebral blood flow patterns in dementia. Electroencephalography and Clinical Neurophysiology, 91, 163-170.

Stevens, A., Kircher, T., Nickola, M., Bartels, M., Rosellen, N., \& Wormstall, H. (2001). Dynamic regulation of EEG power and coherence is lost early and globally in probable DAT. European Archives of Psychiatry and Clinical Neuroscience, 251, 199-204.

Thatcher, R. W., Krause, P. J., \& Hrybyk, M. (1986). Cortico-cortical associations and EEG coherence: A two-compartmental model. Electroencephalography and Clinical Neurophysiology, 64, 123-143.

van Leeuwen, W. S., Wieneke, G., Spoelstra, P., \& Versteeg, H. (1978). Lack of bilateral coherence of mu rhythm. Electroencephalography and Clinical Neurophysiology, 44, 140-146.

Vermersch, P., Scheltens, P., Barkhof, F., Steinling, M., \& Leys, D. (1994). Evidence for atrophy of the corpus callosum in Alzheimer's disease. European Neurology, 34, 83-86.

Wada, Y., Nanbu, Y., Kikuchi, M., Koshino, Y., Hashimoto, T., \& Yamaguchi, N. (1998). Abnormal functional connectivity in Alzheimer's disease: Intrahemispheric EEG coherence during rest and photic stimulation. European Archives of Psychiatry and Clinical Neuroscience, 248, 203-208.

Wada, Y., Nanbu, Y., Koshino, Y., Yamaguchi, N., \& Hashimoto, T. (1998). Reduced interhemispheric EEG coherence in Alzheimer disease: Analysis during rest and photic stimulation. Alzheimer Disease and Associated Disorders, 12, 175-181.

Yesavage, J. A., Brink, T. L., Rose, T. L., Lum, O., Huang, V., Adey, M., \& Leirer, V. O. (1982). Development and validation of a geriatric depression screening scale: A preliminary report. Journal of Psychiatric Research, 17, 37-49.

Received December 1, 2003 Revision received February 17, 2004 Accepted March 1, 2004 